\title{
Optical Signatures of Reprocessing from Hercules X-1
}

\author{
M. D. Still
}

Physics \& Astronomy, University of St. Andrews, Fife KY16 9SS, U.K.

A. P. Reynolds

Sterrekundig Instituut, University of Utrecht, The Netherlands

H. Quaintrell and P. Roche

Astronomy Centre, University of Sussex, Brighton BN1 9QH, U.K.

D. Chakrabarty

Centre for Space Research, MIT, Cambridge, Massachusetts, U.S.A.

S. E. Levine

Observatorio Astronómico Nacional, San Ysidro CA, U.S.A.

\begin{abstract}
.
The mechanism providing the 35-day period found in optical and $\mathrm{X}$-ray light curves of the eclipsing low-mass X-ray binary Her X-1 is considered to be the precession of a tilted and twisted accretion disc about the neutron star. We have obtained spectrophotometric observations of this system. The majority of line absorption and emission originates on or near the mass-losing primary, with distributions over the inner face of the star consistent with irradiation from the environs of the compact object. After suitable corrections for heating, the neutron star is found have a mass of $1.6 \pm 0.3 \mathrm{M}_{\odot}$, in contrast to the last radial velocity study which found a value less than $1.0 \mathrm{M}_{\odot}$. Rotational velocity measurements, while insufficiently precise to reject the older mass estimate, suggest that the rotational period of $\mathrm{HZ}$ Her differs from the synchronous rate by no more than $20 \%$. Using a recently determined figure for the effective temperature of the unheated face, the distance to Her X-1 is estimated at $6.7 \pm 0.4 \mathrm{kpc}$, higher than prior estimates. The systemic velocity is found to be $-69 \pm 6 \mathrm{~km} \mathrm{~s}^{-1}$. In order to to determine the plausibility of the twisted disc model we reconstruct the expected shadow of the disc across the heated inner face of the donor star to compare with observation. Large scale emission structure is reproduced, but the model fails to synthesize finer observational details. HeII $\lambda 4686 \AA$ emission is detected and found to be distributed closely about the neutron star, and is a likely tracer of the accretion flow.
\end{abstract}

